\title{
Image Quality Studying based on lightness
}

\author{
Zaid mohammed abdul kaleq \\ Departments of pharmaceutical, Baghdad college of pharmacy/syndicate of iraq pharmacist, iraq
}

\begin{abstract}
The distortion which occur in the image affects mostly its included information, decreases its sharpness and decreases its contrast. Hence, this leads to overlap of details of different regions and then the spatial resolution of the image decreases. Image quality may be controlled too far by using focusing in accordance to the object to be image and light exposure time of the film or the sensor in the camera. these characteristics have many parameters which may be controlled in order to have good image with high sharpness. Most important details of the image are included in edges regions, but most standards of image estimation don't depend upon specifying the edges in the image and measuring their edges, but they depend upon the general estimation or estimating the uniform region, so we dealt, in our study, with image estimation depending upon calculating the contrast of edges regions. Specifying the edges in the image is made by using soble operator. Also, several different and new methods were proposed to calculate the contrast. Contrast is one of the most standards of the checking image quality.
\end{abstract}

Keywords-exposure of camera, contrast of image, measure of image quality .

\section{Introduction}

For image processing and image contrast enhancement we use the contrast and illumination control process to obtain images with good details and acuity.The image quality in ant optical system depend upon the contrast rate ,brightness ,resolution, optical modulation transfer function. The image quality may be defined as the amount of acuity or contrast in the image [1] . Image quality may be expressed by image resolution, ability to analyze it or its resolution level which may be identified as the ability of optical system to separate the convergent objects and to discriminate them or it is the magnitude of minimum angular separation between two objects in such a manner that each of them is discriminated and separated from other. Also, image quality may be defined as the amount of image sharpness or contrast, since image quality and optical system efficiency are evaluated with several procedures such as point spread function. The optical system with narrow point spread function is better. Human being in most image processing techniques is usually the final judge for image quality after processing. So, using quantitative standards "Signal To Noise Ratio" (SNR) that adopt the error square or any other standards is not exact in examining and sensing the quality of the image[2]. Therefore, it is convenient to use human visual system (HVS) in examining and comparing image quality techniques. Image quality depends upon the spatial resolution, radiometric resolution and spectral resolution. Many former studies concerned with the illumination and contrast effect on the image quality:

$\$$ R. P. Johnson used on 1990 the contrast procedure in detecting edges exist in image with unequal and little lighting. He used domain windows with scaling factors and dimensions of $(5 \times 5)$ in order to detect the edges [ 3 * Scharff L. and Ahumada A submitted, on 2003, they search of possibility of measuring

contrast of texts and possibility of reading them. They examined different conditions of adding the transparent text by summation and multiplying method of texture background. They found that the possibility of word recognition and sort is highly affected by their background [4] .

\section{Measurements of Image contrast}

Contrast is the rate between object lighting and background lighting that the objects fall on. The contrast sensing depends upon the domain distribution of light and dark regions in image. Images would be enhanced by using this characteristics, since a filter may be used to enhance contrast rate in image by subtracting a specific rate from the value of each color unit in image to increase the contrast between image points. Figure(1) shows human Contrast Sensitivity Function (CSF) in human, which depends upon luminance and domain distribution of color in image. The more increased frequency is, the less ability of eye to discriminate $[5,6]$.

\section{The contrast measurements are as follows:}

\section{1 -Weber Contrast measurement}

Weber contrast measurement is one of the oldest measurements of light contrast. It is often used for the forms: small objects with sharp edges like symbols, text symbols over similar backgrounds which are greater than the symbols. Weber Contrast is expressed by this mathematical form, 


$$
C_{w}=\frac{\mathbf{I}_{s}-\mathbf{I}_{b}}{\mathbf{I}_{b}} \text { (1) }
$$

Where $\mathrm{C}_{\mathrm{w}}$ refers to Weber Contrast, $\mathrm{I}_{\mathrm{s}}, \mathrm{I}_{\mathrm{b}}$ refer to symbol and background Lighting respectively [ 5,6 ].

\section{2- Michelson Contrast Measurement}

This measurement is applied to image of pattern like image textures. Michelson Contrast is measured by using the following relation $[3,4]$ :

$$
C_{M}=\frac{\mathrm{I}_{\text {max }}-\mathrm{I}_{\text {min }}}{\mathrm{I}_{\text {max }}+\mathrm{I}_{\text {min }}}
$$

Where $\mathrm{I}_{\max }$ maximum value of Lighting .

$I_{\min }$ minimum value of Lighting .

3.Glare is a sensation resulted from lighting within the optical field which is, practically, higher than the normal lighting that may the eyes adapt to. This makes disturbance or loss in vision efficiency or fine optical performance. Glare is either direct or indirect. Direct glare is represented by sun or electrical lamps which are sources of light. Indirect glare is represented by bright or glossy surfaces like mirrors, glass, and lustrous surfaces as well as surfaces reflecting light like sun and lamps with an angle opposite to vision direction. These two types of glare cause difficulty in vision, distortion to the visual scene and non-relief [ 5 ] .

4. Lighting Distribution is measured from one point to another through a board or a surface which sun ray falls on. Good vision is made when the lighted surface is in proper level of lighting, whereas non-relief vision is on the contrary and may cause harm to the eye due to applied pressure and fast sensing of its lens [ 7 ] .

5. Optimal lighting means insuring the light standard with (lux) which is necessary to perform the required optical tasks in relief, exactly and without any stress to eye. Its amount depends upon the exactness and difficulty of task, it is proportional directly with age of observer and inversely with threshold distance and type of used light. Also, it depends upon surface reflectivity which should be of high reflectivity to produce uniform indirect efficient light, increasing form the source with light unseen to the viewer.

6.Imaging system components See the figure(2)

6.1. Video camera "Sony Video Lens" .

6.2.Photo Transistor Detector ( for reflecting lightness intensity measurement ), whereas lightness intensity unit is "lux".

6.3. Voltmeter and Ammeter ( for measuring applied voltage and passing current and determining power by multiplying voltage by current), whereas power unit is "power".

6.4.Tungsten lamp (light source), which Emitting wave lengths slightly differ from the wave lengths of sun light

Several images were taken for different tungsten lightings by digital camera and recording process were made as video clips, time of each clip is 1.5 minute, they are still images. These video clips were transmitted to a computer after being cut and regulated according to value of voltage applied to the imaging system using the software "Ulead Video Studio 9" and stored as separated images with Bitmap and represented with 24 bit. Hence, we obtained 14 images for different tungsten lightings for white sheet image and experimental image "color bar" respectively, as shown in figure( 3 )and figure ( 4 ).

\section{Results of Extract line from test White image}

To study the distribution of lighting intensity has been extract line in the half width and half height of chromatic homogeneous (white) image taken in different light intensities. To study the statistical characteristics the mean and standard deviation of RGBL intensity of the extracted line. Relation between the mean $(\mu)$ and standard deviation $(\sigma)$ of chromatic composites is shown in figure (7) for coordinate $\mathrm{x}$ and (8) for coordinate $\mathrm{y}$. When the distance between light source and scene to be imaged is $46 \mathrm{~cm}$, the relation will be linear and direct to a specific extent, i.e. ( $0-180)$ volt range which corresponds to mean $(\mu)$ of $(0-160)$ volt , then it becomes like a curve in voltage range of (200-250) volt. Increase in mean $(\mu)$ and standard deviation $(\sigma)$ indicate an increase in resolution in the image which depends upon light, since both values indicate an increase in resolution and after reaching to a specific extent, lightness in the image will increase at the expense of contrast of the image .

\section{Contrast measuring results}

In this study several techniques were suggested based on edges regions among to find image contrast So, contrast is calculated for chromatic composites RGB and lighting composites L as follows: 


\section{1- Direct Contract Technique}

This technique using contrast equation ( 2 ) and applying to image edges points regions only. This would be made by taking triple windows around edges points and searching for lower and higher value in intensity of image components relating to edges .

\section{2-Contrast measuring based on statistical characteristics}

In this technique statistical characteristics of edges points in image for contrast calculation were adopted according to equation (2). $I_{\min }$ and $I_{\max }$ are calculated by adopting the mean $(\mu)$ and standard deviation $(\sigma)$ of edges regions according to the two following relations:

$\mathbf{I}_{\min }=\mu-\sigma \quad$ and $\quad \mathrm{I}_{\max }=\mu+\sigma \quad, \quad \therefore \mathrm{c}=\frac{\mu}{\sigma}$ may contrast measure (3)

Contrast techniques are applied to edges regions only where contrast in edge regions is calculated after specifying the edges using Soble operator. Thereafter, contrast is specified by taking $3 \times 3$ size window around each edge component. The direct contrast is calculated by applying the relation (2), contrast adopting the statistical characteristics is calculated from equation (3). Contrast was calculated with different thresholds of Soble operator i.e. 20,40,60, 80 and 100. After calculating contrast for each image with different lightness, power of lighting system is calculated by multiplying current by applied voltage. Relation between mean contrast and power for Direct Contrast Technique is illustrated in figure (8), Contrast Technique adopting the Statistical Characteristics of imaging system is illustrated in figure (9) Through the suggested techniques of contrast calculation optimal image with high contrast for lighting system with tungsten lamp was identified, i.e. get optimal image at illumination power 36 watt.

\section{Figures}

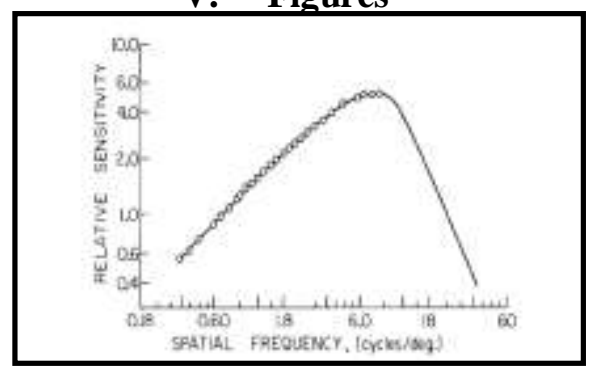

Figure ( 1 ) : Contrast Sensitivity Function of Domain Frequency Function [ 1]

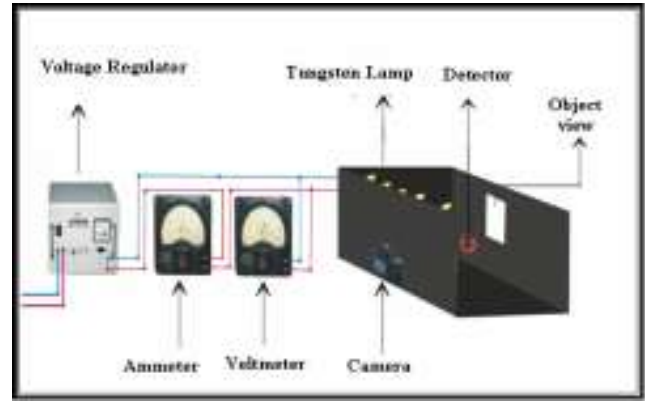

Figure 2: Imaging System by using Tungsten filament lamp


Figure 3: White sheet experimental images for different tungsten lightings 


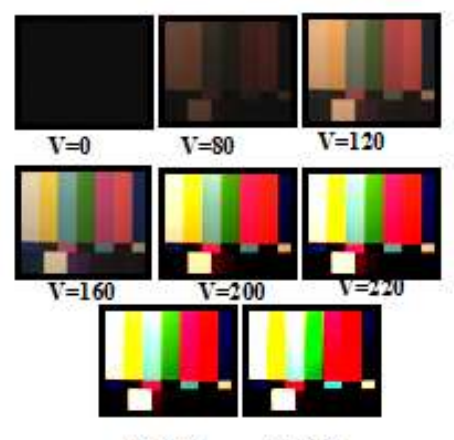

Figure 4: Color bar experimental images for different tungsten lightings
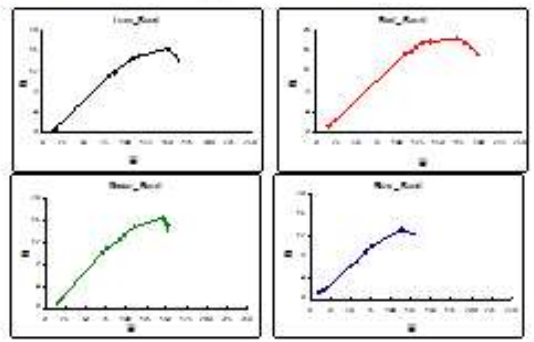

Figure 6: Relation between the mean $(\mu)$ and standard deviation $(\square)$ of chromatic composites and light composite Coordinate $x$
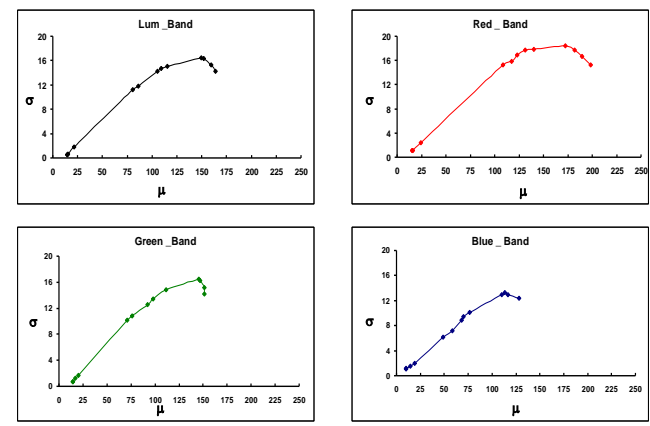

Figure 7: Relation between the mean $(\mu)$ and standard deviation $(\sigma)$ of chromatic composites and light composite Coordinate y
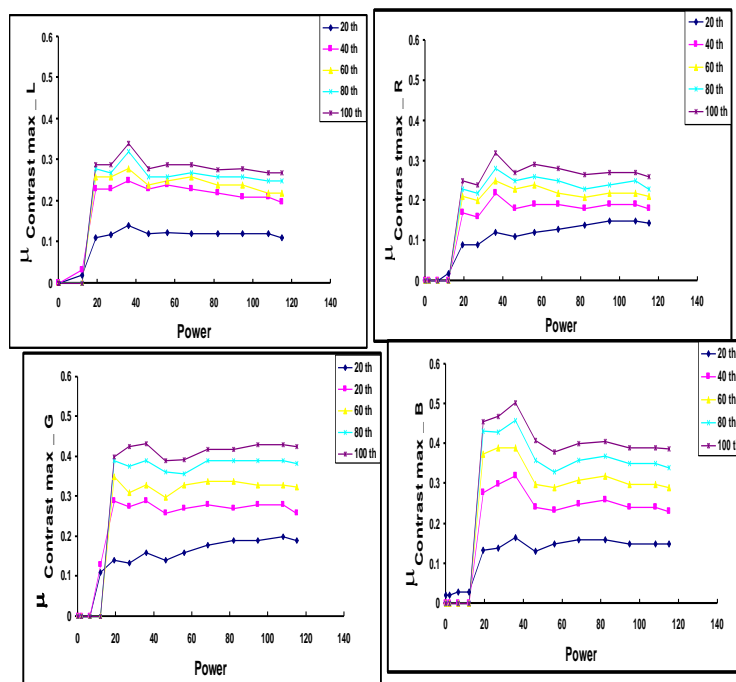

Figure (8) Direct Contract Technique (RGBL) for different tungsten lightings with different threshold of soble operator 

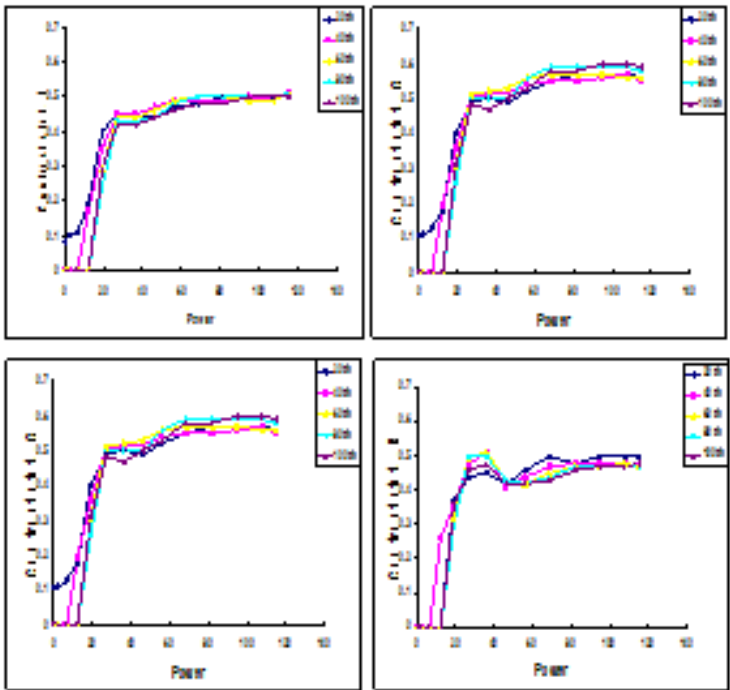

Figure (9) Contrast measure based on statistical characteristics for different tungsten lightings

\section{References}

[1] K William. , " Digital Image Processing ", $3^{\text {rd }}$, Inc. ,ISBNS: $0-471-37407-5$ ( Hard back ), $0-471-22132-5$ ( electronic ),(2001).

[2] Y Ramamurthy., , N Naren dran., J Freyssinier .," Determining Contrast Sensitivity function for Monochromatic light emitted By high-Brightness" Third international Conference on solid state, (2004).

[3] R Johnson. , " Contrast Based Edge Detection ", Pattern Recognition, vol.23,no.3/4, (1990 ).

[4] L Scharff . and A Ahumada ., " Contrast Measure For Predicting text readability ", Airspace operation systems (AOS) project of Nasa's Airspace systems program, (2003).

[5] D Lamming., " Contrast Sensitivity" , chapter ( 5 ), in Cronly Dillon, Vision and Visual Dysfunction, vo.15 , London , (1997).

[6] A Roorda . and D Williams., " The Arrangement of three Cone Classes in the Living Human Eye " , Nature 11: 520 - 522 ,( 1999).

[7] J Callender ., " Time - Saver Standards for Architectural Design Data" ,5 th .ed . U.S.A ;Mc, Graw -Hill Inc, 1974 